\title{
Sliding Mode Control with Model-Based Switching Functions applied on a 7-DOF Exoskeleton Arm
}

\author{
Charles Fallaha, Maarouf Saad, Senior Member, IEEE, Jawhar Ghommam, and Yassine Kali
}

\begin{abstract}
This paper features a novel sliding mode controller for robotic arms using nonlinear model-based switching functions. The new controller is experimentally validated on a 7-DOF exoskeleton arm used for upper-limb rehabilitation applications. The proposed approach features a novel concept using modelbased switching functions in the sliding mode controller, which leads to considerable simplifications on the torque control inputs. Compared to conventional linear switching functions, modelbased switching functions show substantial control performance improvements on the torque inputs, such as transient constraints reduction and enhanced robustness, while maintaining a very good tracking performance. Moreover, model-based switching functions design ensures a complete decoupling of chattering effect between joint axes. Furthermore, this approach can be combined with existing chattering reduction techniques to ensure proper control of chattering levels on the torque inputs. These advantages make the practical implementation of the modelbased switching functions approach particularly desirable for wearable robotics, where smooth movements and high accuracy are important requirements for patients' comfort and security.
\end{abstract}

Index Terms-Sliding Mode Control, Exoskeleton, upper-limb, Robot, Model-Based Switching Functions, Chattering, Robust Control.

\section{INTRODUCTION}

$\mathbf{T}$ HE last decade has known a tremendous growth and diversification in robotics systems, which has been mainly supported by recent advances, accessibility and affordability of robotics technology. CPU computing power is increasing, sensors are getting smaller and cheaper, and robotic applications programming is becoming easier and more accessible. By the end of 2025, the global robotics market is expected to reach 87 billion US dollars [1]. The commercial robotics market, including services and healthcare applications, will be valued by then at approximately 23 billion dollars, in which biomedical robotics will take an important part.

Within the biomedical robotics segment, the wearable robotics market, and more specifically exoskeleton devices market, is expected to undergo a steady growth during the next decade. In 2019, the global exoskeleton market was valued at 626.3 million US dollars, in which healthcare exoskeletons took the largest share [2]. This valuation had almost quintupled from 2017 [3]. This is therefore triggering extensive research for exoskeleton design optimization and control.

Upper-limb exoskeletons are a very popular type of exoskeletons that are widely studied in literature. They are mainly used for upper-limb sports injuries or to treat patients

C. Fallaha, M. Saad and Y. Kali are with the Department of Electrical Engineering, École de Technologie Supérieure, Montreal, QC H3C 1K3, Canada e-mail: (charles.fallaha.1@ens.etsmtl.ca).

J. Ghommam is with the Department of Electrical and Computer Engineering, Sultan Quaboos University, Muscat 123, Oman that have undergone stroke incidents resulting in upper-limb impairments. Scientific literature presents various upper-limb exoskeleton structures, control strategies and technologies integration. The authors in [4] have designed a cable-actuated exoskeleton arm. In [5], pneumatic actuators are used for the upper-limb exoskeleton structure to emulate the mechanical and dynamic structure of human upper-limb muscles. Recent advances in virtual reality (VR) and augmented reality (AR) also allow for an effective integration of these technologies for human-in-the-loop exoskeleton controllers [6]. Haptic feedback and dynamic admittance control [7] are also control strategies that give patients realistic interactions and contact sensations and need to follow specific mechanical structures requirements [8]. Biological signals such as measured Electromyogram (EMG) signals can be used as well to control actuator movements of the exoskeleton arm [9].

Although simple linear control strategies such as PIDs can be used for upper limb exoskeleton control [10], they lack overall robustness in practical implementations [11]. This paper proposes a robust nonlinear control strategy study on an experimental upper-limb 7-DOF exoskeleton prototype named Motion Assistive Robotic-Exoskeleton for Superior Extremity (ETS-MARSE) [12]-[15]. ETS-MARSE has been designed to provide effective rehabilitation therapy for patients that have suffered upper-limb impairment and is currently being used for this purpose. ETS-MARSE comprises a shoulder motion support part, an elbow and forearm motion support part, and a wrist motion support part. All these parts are directly driven by electrical actuators and rigid gearing systems.

The nonlinear control strategy of ETS-MARSE presented in this paper is based on the sliding mode control approach, which has been widely used for controlling nonlinear systems. The inherent robustness properties of sliding mode control remove the need of adding adaptive algorithms to the control loop [16]. Sliding mode control can be applied to a wide range of dynamic SISO or MIMO systems and has been recently applied on numerous robotic and mechatronic systems [17][19]. Sliding mode control can also be combined with other nonlinear control techniques for robotic arms control [11].

The main downside of conventional sliding mode control is the chattering phenomenon existing on the control input signals. This chattering effect is due to a discontinuous term used in the construction of the control law input. Numerous approaches have been proposed to address this problem. The boundary layer approach [16] smoothens the discontinuous term by replacing the signum function with a saturation function. [20], [21] and more recently [22] have explored nonlinear reaching laws that reduce the discontinuous gain when the system approaches the sliding surface. The authors in [23] 
have used higher-order sliding mode (HOSM) to eliminate chattering. This approach requires however the implementation of higher order observers in its traditional implementation. Several authors [24]-[26] have explored a particular case of HOSM, namely second-order sliding mode. [27] proposed a third order sliding mode control. More recently, a variant of HOSM, the super-twisting algorithm, has been proposed by [28] and studied further by [29] and [30].

Conventional sliding mode control forces the system's closed loop dynamics to vary on a linear switching surface that guarantees the convergence of the tracking error to 0 . Some developments can be found in literature in which nonlinear switching functions such as Terminal Sliding Mode (TSM) control [31] are used mainly to improve the dynamic performance of the tracking error. This paper introduces therefore a novel concept in sliding mode control applied to robotic systems, whereby traditional linear switching functions are replaced by nonlinear model-based switching functions. Modelbased switching functions substantially simplify the expression of the torque control inputs, while ensuring trajectory tracking asymptotic convergence on the switching surface. This leads to transient constraints reduction on the control input, as well as a complete chattering decoupling on all joint axes. This improvement prevents against premature failures of actuating components in the system, and avoids unaccounted for fast dynamics behaviour in the closed-loop system. Experimental results on ETS-MARSE show that the proposed approach leads to an improved tracking performance with lower torque control inputs variance compared to conventional sliding mode approach. The proposed approach can furthermore be combined with known chattering reduction techniques and optimal control strategies such as Model Predictive Control (MPC) for practical implementation [32] and [11].

To the best knowledge of the authors, the original contributions of this paper are summarized into the following points:

1) A novel approach for designing nonlinear switching functions based on any robotic structure model and focuses on the design of trajectory tracking switching functions. This ensures considerable simplifications on the torques control law, while providing asymptotic stability of the state error. These simplifications lead to a linear relationship of the torque inputs with regards to the joints errors and error rates. This approach reduces control inputs activity while keeping excellent tracking performance and robustness, as shown in the experimental results. Moreover, the proposed approach ensures a complete decoupling of the chattering on all joint axes, which represents an important advantage compared to typical sliding mode control.

2) A novel general expression of a relationship on the gravity term of the robot's dynamic model ( (12), (33)). This relationship leads to the formulation of a stability criterion on the controller's parameters. This relationship is also useful into extending the validation of the robot's dynamic model to the gravity term.

The paper is structured as follows: In section II the ETSMARSE Exoskeleton structure, kinematic and inertial param-

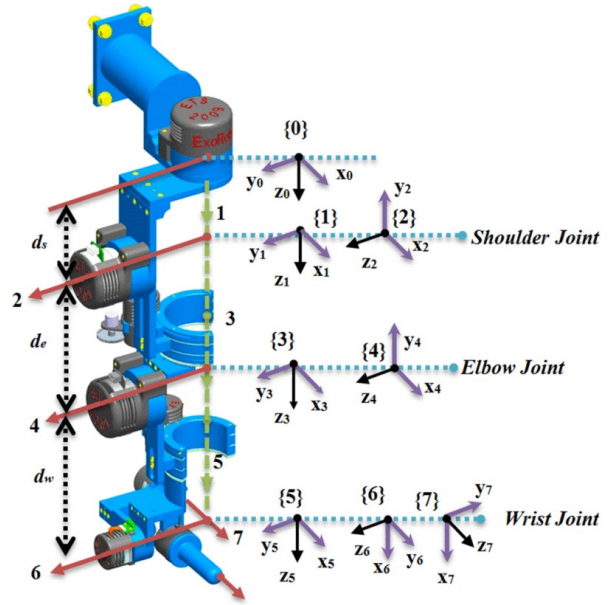

Fig. 1. ETS-MARSE Structure and Reference Frames Assignation

eters are presented. In section III, conventional sliding mode control is applied on robotic systems and the problem statement is formulated. Section IV details model-based switching functions design for both the set point and the trajectory tracking problems and highlights the contributions of the proposed approach. Section V describes the experimental setup of ETS-MARSE and presents real-time results of the proposed approach. Section VI concludes the paper.

\section{ETS-MARSE 7-DOF EXOSKELETON CHARACTERIZATION}

ETS-MARSE is a redundant 7-DOF robot prototype designed to ease upper-limb movement of physically disabled individuals with impaired upper-limb function. ETS-MARSE could be used for rehabilitation therapies of patients who have experienced sports injuries or stroke incidents that left their upper-limbs impaired. Fig. 1 shows the 7 actuation joints with the joint axes assignation following the modified DenavitHartenberg (DH) convention [33]. The correspondent modified DH parameters can be derived as shown in Table I below. The design of ETS-MARSE emulates the anatomy of the human's upper-limb with the known 7 degrees of movement, with the objective of providing the user a representative and ergonomic experience during therapy sessions.

As depicted in Fig. 1, the shoulder motion consists of three joints, the elbow motion comprises one joint, and finally the wrist motion consists of three joints.

TABLE I

MODIFIED DH PARAMETERS OF ETS-MARSE

\begin{tabular}{ccccc}
\hline \hline Joint $(i)$ & $\alpha_{i-1}(\mathrm{rad})$ & $a_{i-1}(\mathrm{~m})$ & $d_{i}(\mathrm{~m})$ & $\theta_{i}(\mathrm{rad})$ \\
\hline 1 & 0 & 0 & $d_{s}$ & $\theta_{1}$ \\
2 & $-\pi / 2$ & 0 & 0 & $\theta_{2}$ \\
3 & $\pi / 2$ & 0 & $d_{e}$ & $\theta_{3}$ \\
4 & $-\pi / 2$ & 0 & 0 & $\theta_{4}$ \\
5 & $\pi / 2$ & 0 & $d_{w}$ & $\theta_{5}$ \\
6 & $-\pi / 2$ & 0 & 0 & $\theta_{6}-\pi / 2$ \\
7 & $-\pi / 2$ & 0 & 0 & $\theta_{7}$ \\
\hline \hline
\end{tabular}


TABLE II

PHYSICAL WORKSPACE LIMITS OF ETS-MARSE

\begin{tabular}{ccc}
\hline \hline Joint $(i)$ & Motion & Workspace \\
\hline 1 & Shoulder joint horizontal flexion/extension & $0^{\circ} / 140^{\circ}$ \\
2 & Shoulder joint vertical flexion/extension & $140^{\circ} / 0^{\circ}$ \\
3 & Shoulder joint internal/external rotation & $-85^{\circ} / 75^{\circ}$ \\
4 & Elbow joint flexion/extension & $120^{\circ} / 0^{\circ}$ \\
5 & Forearm joint pronation/supination & $-85^{\circ} / 85^{\circ}$ \\
6 & Wrist joint ulnar/radial deviation & $-30^{\circ} / 20^{\circ}$ \\
7 & Wrist joint flexion/extension & $-50^{\circ} / 60^{\circ}$ \\
\hline \hline
\end{tabular}

TABLE III

INERTIAL PARAMETERS OF ETS-MARSE

\begin{tabular}{cccc}
\hline \hline Joint $(i)$ & Mass $(\mathrm{kg})$ & Center of mass $(\mathrm{m})$ & Link length $(\mathrm{m})$ \\
\hline 1 & 3.475 & 0.0984 & 0.145 \\
2 & 3.737 & 0.1959 & 0 \\
3 & 0 & 0 & 0.25 \\
4 & 2.066 & 0.163 & 0 \\
5 & 0 & 0 & 0.267 \\
6 & 0.779 & 0.121 & 0 \\
7 & 0.496 & 0.0622 & 0 \\
\hline \hline
\end{tabular}

Table II characterizes the total workspace of ETS-MARSE by detailing the motion intervals of each joint. ETS-MARSE has a relatively small footprint structure and a remarkably high-power density with regards to weight, which considerably eases its installation, handling and operation. The measured weight and inertial parameters of ETS-MARSE are displayed in Table III. ETS-MARSE can operate in passive motion mode (supports entirely the subject's upper-limb motion) and in active motion mode (uses force sensors [13], [15], or EMG [14] feedback signals to accompany and assist the subject's upper-limb motion).

\section{Nonlinear Control Design for ETS-MARSE}

The control design for ETS-MARSE is based on the sliding mode control approach which forces the dynamics of the closed-loop system to remain or "slide" on a linear surface $S=0$. Fig. 2 depicts the process in the phase plane for the case of a two-dimensional state vector system. The sliding mode control process includes two phases. The first phase, also referred to as the reaching phase, is ensured by a discontinuous control term $\left(u_{d i s c}\right)$ that allows the state error to reach the sliding surface. The second phase, or sliding phase, is ensured by a continuous equivalent term $\left(u_{e q}\right)$ and keeps the error dynamics on the sliding surface and ultimately leads the error vector towards to the zero-equilibrium point [22].

\section{A. Conventional Sliding Mode Control Applied to Robotic Systems}

The general free dynamics of a multi-joint friction less robot without external constraints follows the nonlinear second-order differential equation in the joint angle space of the robot [33]:

$$
\tau=M(\theta) \ddot{\theta}+V_{m}(\theta, \dot{\theta}) \dot{\theta}+G(\theta)
$$

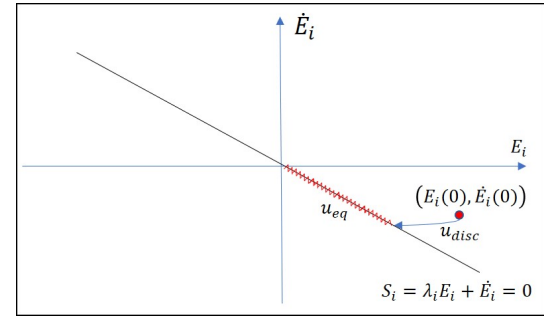

Fig. 2. Asymptotic Convergence on a linear sliding surface depicted in the phase plane

where $\tau \in R^{n}$ is the torque control input vector, $\theta \in R^{n}$ is the robot joint angles and/or displacements vector, $M(\theta) \in R^{n \times n}$ is the inertia matrix, and is positive definite, $V_{m}(\theta, \dot{\theta}) \in R^{n \times n}$ is the centrifugal and Coriolis matrix and $G(\theta) \in R^{n}$ is the gravity term vector.

With conventional sliding mode control associated with second-order MIMO systems, a typical first order linear switching function vector is chosen in terms of the tracking error and its time derivative:

$$
S=\Lambda E+\dot{E}, \Lambda=\operatorname{diag}\left(\lambda_{i}\right), \lambda_{i}>0
$$

where $E=\theta-\theta_{R}$ is the state tracking error vector defined as the difference between the measured joint angles/displacements and reference joint angles/displacements vector. The torque control law is then designed to force the tracking error vector $E$ to reach and stay on the surface $S=0$. As per (2), this leads the error vector to reach the zeroequilibrium point following first order convergence dynamics. The convergence rate of each differential equation is controlled by the values of $\lambda_{i}$. To ensure $S=0$ is reached, the control law is designed to practically force the following relationship, often referred to as reaching law:

$$
\dot{S}=-K \operatorname{sign}(S), K=\operatorname{diag}\left(k_{i}\right), k_{i}>0
$$

where $\operatorname{sign}(S)=\left[\operatorname{sign}\left(S_{1}\right), \cdots, \operatorname{sign}\left(S_{n}\right)\right]^{T}$ with $\operatorname{sign}\left(S_{i}\right)$ for $i=1, \cdots, n$ is defined as follows:

$$
\operatorname{sign}\left(S_{i}\right)=\left\{\begin{array}{cl}
1, & \text { if } S_{i}>0 \\
-1, & \text { if } S_{i}<0 \\
0, & \text { if } S_{i}=0
\end{array}\right.
$$

Equation (3) allows the error state vector to reach the surface $S=0$ in a finite time that is dependent upon the values of $k_{i}$. Substituting (1) and the time derivative of (2) in (3) gives the following torque vector control law:

$\tau=\underbrace{V_{m}(\theta, \dot{\theta}) \dot{\theta}+G(\theta)-M(\theta)\left(\Lambda \dot{E}-\ddot{\theta}_{R}\right)}_{u_{e q}}-\underbrace{M(\theta) K \operatorname{sign}(S)}_{u_{\text {disc }}}$

Control law (5) is composed of the two terms $u_{e q}$ and $u_{\text {disc }}$ depicted in Fig. 2.

\section{B. Problem Statement}

From (5), the expression of $u_{e q}$ and $u_{d i s c}$ is complex and highly nonlinear. This complexity leads to transient and steady-state constraints on the torque control inputs. $u_{e q}$ contains the robot's dynamic matrices for model compensation. 


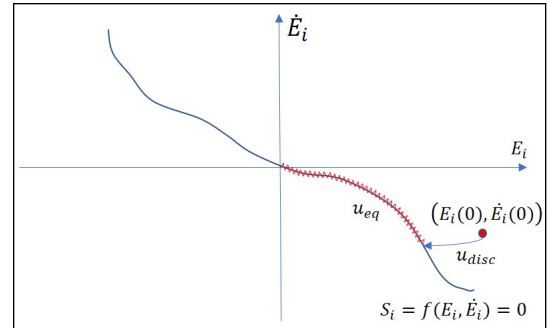

Fig. 3. Asymptotic convergence with model-based switching functions in the phase plane

For fast trajectory tracking applications, this implies higher dynamic constraints on the global torque control law. Analog or digital noise from sensing systems are also amplified through these nonlinear matrices. On the other hand, the discontinuous term $u_{\text {disc }}$ includes the robot's inertia matrix that multiplies the signum term $\operatorname{sign}(S)$. In the general case, $M(\theta)$ is a non-constant and non-diagonal positive definite matrix that leads to a coupling effect between torque input signals when a chattering condition exists on one or more axes. Moreover, the inertia matrix has a direct modulating impact on the chattering amplitudes on the torque input signals. Control law (5) can be simplified by transferring model-based terms from the equivalent torque model-compensation term $u_{e q}$ into the switching functions inside the discontinuous term $u_{d i s c}$. The resultant switching functions become nonlinear, modeldependent and coupled differential functions. Fig. 3 depicts such nonlinear switching functions in the phase plane.

\section{Model-Based Switching Functions Design APPLIED TO ROBOTIC MANIPULATORS}

This section details the design of model-based switching functions for any robot arm, and can be generalized to any second order mechanical system which dynamic differential equations can be formalized into relationship (1). The corresponding sliding surfaces are proved to be asymptotically stable, and the torque control law is shown to have a much simpler structure compared to (5). This approach is first introduced for a setpoint convergence problem, and its main results are summarily covered in sub-section A below. This sets the framework basis for the generalized tracking error convergence problem covered in sub-section B.

\section{A. Model-Based Switching Functions Design for the Zero Setpoint Convergence Problem}

For zero setpoint convergence using sliding mode control, consider the following switching function [34]:

$$
\begin{aligned}
S(\theta, \dot{\theta})= & M(\theta) \dot{\theta}+\Gamma \theta+\Xi \int_{t_{0}}^{t} \theta d t+\int_{t_{0}}^{t}(G(\theta)-G(0)) d t \\
& -\int_{t_{0}}^{t}\left(\sigma(\theta, \dot{\theta})+V_{m}(\theta, \dot{\theta})\right) \dot{\theta} d t
\end{aligned}
$$

where $\sigma(\theta, \dot{\theta})$ is a skew-symmetric matrix binding matrix $M(\theta)$ and $V_{m}(\theta, \dot{\theta})$ into the following well-known relationship [35]:

$$
\sigma(\theta, \dot{\theta})=\dot{M}(\theta)-2 V_{m}(\theta, \dot{\theta})
$$

$\Gamma$ and $\Xi$ are symmetrical positive definite constant matrices chosen to achieve the required dynamics performance when the system reaches the sliding surface $S=0$. Note from (6) that $S(\theta, \dot{\theta})$ has integral terms that can directly place the system on the sliding surface with adequate choice of initial conditions of the integrators. The time derivative of $S(\theta, \dot{\theta})$ can be deduced from (6):

$$
\begin{aligned}
\dot{S}(\theta, \dot{\theta})= & M(\theta) \ddot{\theta}+\dot{M}(\theta) \dot{\theta}+\Gamma \dot{\theta}+\Xi \theta+G(\theta)-G(0) \\
& -\left(\sigma(\theta, \dot{\theta})+V_{m}(\theta, \dot{\theta})\right) \dot{\theta}
\end{aligned}
$$

Using (7) in (8) gives the following:

$$
\dot{S}(\theta, \dot{\theta})=M(\theta) \ddot{\theta}+V_{m}(\theta, \dot{\theta}) \dot{\theta}+\Gamma \dot{\theta}+\Xi \theta+G(\theta)-G(0)
$$

Using (1) in (9):

$$
\dot{S}(\theta, \dot{\theta})=\tau+\Gamma \dot{\theta}+\Xi \theta-G(0)
$$

Therefore from (10) the torque control law that ensures the reaching law (3) is:

$$
\tau=\underbrace{-\Gamma \dot{\theta}-\Xi \theta+G(0)}_{u_{e q}}-\underbrace{K \operatorname{sign}(S(\theta, \dot{\theta}))}_{u_{d i s c}}
$$

Remark 1: Compared to the torque control law (5) given by conventional sliding mode control, (11) shows a much simpler torque control input structure with simplified expressions $u_{e q}$ and $u_{\text {disc }}$. Control law (11) shows moreover the torque inputs are linear in terms of joint angles and rates with a decoupling of the chattering effect on the axes, provided that $K$ is chosen to be a diagonal matrix.

The next step is to prove that the sliding surface $S(\theta, \dot{\theta})=0$ ensures asymptotic convergence of the joint angles towards the zero-equilibrium point. This is shown in proposition 1 below and uses the following lemma [34].

Lemma 1: The difference $G(\theta)-G(0)$ can be written as:

$$
G(\theta)-G(0)=-\Psi_{G}(\theta) \theta
$$

where $\Psi_{G}(\theta)$ is a symmetric matrix defined as follows:

$$
\Psi_{G}(\theta)=-\int_{0}^{1} J_{G}(h . \theta) d h
$$

where $J_{G}$ is the square Jacobian of $G$ defined as follows:

$$
J_{G}=\left[\frac{\partial G_{i}}{\partial \theta_{j}}\right]
$$

Proof: Using the mean value theorem applied to differentiable vector functions, the following holds [36]:

$$
G(\theta)-G(0)=\left(\int_{0}^{1} J_{G}(h . \theta) d h\right) \theta
$$

where $J_{G}$ is the square Jacobian of $G$ defined by (14).

Using the fact that $G$ is derived from a potential energy $U$, namely:

$$
G_{i}=\frac{\partial U}{\partial \theta_{i}}
$$

Then the Jacobian matrix can be written as:

$$
J_{G}=\left[\frac{\partial}{\partial \theta_{j}} \frac{\partial}{\partial \theta_{i}} U\right]
$$


Under the assumption that the second partial derivatives of $U$ are continuous, then Schwarz' theorem yields the following:

$$
\frac{\partial}{\partial \theta_{j}} \frac{\partial}{\partial \theta_{i}} U=\frac{\partial}{\partial \theta_{i}} \frac{\partial}{\partial \theta_{j}} U
$$

Therefore $J_{G}$ is symmetric.

Noting $\Psi_{G}(\theta)=-\int_{0}^{1} J_{G}(h . \theta) d h, \Psi_{G}(\theta)$ is also symmetric as it is the integral of a symmetric matrix, which completes the proof of Lemma1. Note that since $\Psi_{G}(\theta)$ is symmetric, it is diagonalizable, and all its eigenvalues are real.

Proposition 1: [34], the sliding surface $S(\theta, \dot{\theta})=0$, where $S(\theta, \dot{\theta})$ is given by (6), ensures the asymptotic stability of $\theta$ to 0 provided that the eigenvalues of matrix $\Xi$ verify the following constraint:

$$
\operatorname{Min}_{i}\left(\operatorname{Eig}_{i} \Xi\right)>\operatorname{Max}_{i}\left(\operatorname{Eig}_{i} \Psi_{G}(\theta)\right)
$$

Proof: Relationship (19) implies that the difference $\Xi-$ $\Psi_{G}(\theta)$ is positive definite. When the sliding surface $S(\theta, \dot{\theta})=$ 0 is reached, (11) becomes:

$$
\tau=-\Gamma \dot{\theta}-\Xi \theta+G(0)
$$

Consider now the following Lyapunov candidate function:

$$
L(\theta, \dot{\theta})=\frac{1}{2} \dot{\theta}^{T} M(\theta) \dot{\theta}+P(\theta)-P(0)
$$

with $P$ a scalar function defined as [37]:

$$
P(\theta)=U(\theta)+\frac{1}{2} \theta^{T} \Xi \theta-\theta^{T} G(0)
$$

$U$ is the potential energy term from which is derived $G(\theta)$. Differentiating $P(\theta)$ with respect to $\theta$ and noting that $\frac{\partial U(\theta)}{\partial \theta}=G(\theta)$ [35], then using Lemma 1, the following relation can be obtained:

$$
\frac{\partial P(\theta)}{\partial \theta}=G(\theta)+\Xi \theta-G(0)=\left(\Xi-\Psi_{G}(\theta)\right) \theta
$$

Since $\Xi-\Psi_{G}(\theta)$ is positive definite, then $\frac{\partial P(\theta)}{\partial \theta}=0$ only for $\theta=0$, and therefore $P(\theta)$ is absolute minimum for $\theta=0$ [37], which implies $P(\theta)-P(0)>0$ for $\theta \neq 0$. Thus, from (21) it can be deduced that $L(\theta, \dot{\theta})$ is a Lyapunov function. Differentiating $L(\theta, \dot{\theta})$ with respect to time gives:

$\dot{L}(\theta, \dot{\theta})=\dot{\theta}^{T} M(\theta) \ddot{\theta}+\frac{1}{2} \dot{\theta}^{T} \dot{M}(\theta) \dot{\theta}+\dot{\theta}^{T} G(\theta)+\dot{\theta}^{T} \Xi \theta-\dot{\theta}^{T} G(0)$

The above equation can also be written as:

$$
\dot{L}(\theta, \dot{\theta})=\dot{\theta}^{T} \tau+\dot{\theta}^{T} \Xi \theta-\dot{\theta}^{T} G(0)
$$

Using relationship (20), (25) simplifies into:

$$
\dot{L}(\theta, \dot{\theta})=-\dot{\theta}^{T} \Gamma \dot{\theta}
$$

Therefore $\dot{L}(\theta, \dot{\theta})$ is negative semi-definite. Applying Barbalat's lemma [16], $\dot{\theta}$ converges to 0 . Using (1) and (20) it comes that:

$$
\Xi \theta+(G(\theta)-G(0))=\left(\Xi-\Psi_{G}(\theta)\right) \theta \underset{\mathrm{t}}{\rightarrow} 0
$$

Since $\Xi-\Psi_{G}(\theta)$ is positive definite, this implies that $\theta$ converges to 0 , which completes the proof of Proposition 1.
Remark 2: Note that one can choose to compensate the gravity term in the torque control law rather than in the switching functions. In this case, constraint (19) is not necessary anymore, and the switching function (6) becomes:

$S(\theta, \dot{\theta})=M(\theta) \dot{\theta}+\Gamma \theta+\Xi \int_{t_{0}}^{t} \theta d t-\int_{t_{0}}^{t}\left(\sigma(\theta, \dot{\theta})+V_{m}(\theta, \dot{\theta})\right) \dot{\theta} d t$

However, the torque control law becomes:

$$
\tau=-\Gamma \dot{\theta}-\Xi \theta+G(\theta)-K \operatorname{sign}(S(\theta, \dot{\theta}))
$$

\section{B. Switching Functions Design for the Trajectory Tracking Problem}

Trajectory tracking development in the robot's joint space is a generalization of the above set point development whereby model-based switching functions are designed such that the error $E=\theta-\theta_{R}$ asymptotically converges to 0 . Consider the following model-based sliding function for the trajectory tracking problem:

$$
\Sigma=S(\theta, \dot{\theta})-S\left(\theta_{R}, \dot{\theta}_{R}\right)
$$

Where $S$ is defined by (6).

Remark 3: Note that the design of switching function $\Sigma$ defined by (30) is not equivalent to replacing $\theta$ with the tracking error $E$ in (6) and forming $S(E, \dot{E})$, since $S$ is a nonlinear function. This specific choice of $\Sigma$ ensures similar levels of simplifications of the torques control inputs as for the set-point tracking problem, and therefore is completely novel and remains different from the set-point convergence problem in [34].

It can be shown that the torque control law that allows the system to reach $\Sigma=0$ is given by:

$$
\tau=\underbrace{\tau_{R}-\Gamma \dot{E}-\Xi E}_{u_{e q}}-\underbrace{K \operatorname{sign}(\Sigma)}_{u_{\text {disc }}}
$$

where

$$
\tau_{R}=M\left(\theta_{R}\right) \ddot{\theta}_{R}+V_{m}\left(\theta_{R}, \dot{\theta}_{R}\right) \dot{\theta}_{R}+G\left(\theta_{R}\right)
$$

Note that $\tau_{R}$ is exclusively composed of constructed reference signals and doesn't include any measured or estimated signal. Fig. 4 below shows the block diagram of the proposed general control algorithm with model-based switching functions design. This block diagram shows that the control strategy simplifies essentially to a proportional and derivative controller for the feedback loop, with a feedforward term $\tau_{R}$ solely dependent upon the reference, and the robust switching signum term that ensures the reaching condition on the model-based switching surfaces.

Remark 4: The torque control law (31) shows again that the torque inputs become linear in terms of the error vector and its time derivative when using model-based switching functions. Note again from (31) the discontinuity decoupling between joint axes. It remains to prove that $\Sigma=0$ ensures asymptotic convergence of the error vector $E$ towards 0 . Similarly to the set point approach, consider the following lemma.

Lemma 2: The difference $G(\theta)-G\left(\theta_{R}\right)$ can be written as:

$$
G(\theta)-G\left(\theta_{R}\right)=-\Psi_{G}\left(\theta, \theta_{R}\right)\left(\theta-\theta_{R}\right)
$$


Where $\Psi_{G}\left(\theta, \theta_{R}\right)$ is a symmetric matrix defined as follows:

$$
\Psi_{G}\left(\theta, \theta_{R}\right)=-\int_{0}^{1} J_{G}\left(\theta_{R}+h\left(\theta-\theta_{R}\right)\right) d h
$$

where $J_{G}$ is the same square Jacobian of $G$ defined by (14). The proof of Lemma 2 is closely based on that of Lemma 1.

Proposition 2: The sliding surface $\Sigma=0$ where $\Sigma$ is given by (30), ensures the asymptotic convergence of $\theta$ to $\theta_{R}$ provided that the eigenvalues of matrix $\Xi$ verify the following constraint:

$$
\operatorname{Min}_{i}\left(\operatorname{Eig}_{i} \Xi\right)>\operatorname{Max}_{i}\left(\operatorname{Eig}_{i} \Psi_{G}\left(\theta, \theta_{R}\right)\right)
$$

Proof: Relationship (35) implies that the difference $\Xi-$ $\Psi_{G}\left(\theta, \theta_{R}\right)$ is positive definite. When $\Sigma=0$, (31) leads to the following:

$$
\tau=\tau_{R}-\Gamma \dot{E}-\Xi E
$$

Consider now the following Lyapunov-like candidate function:

$$
L=\frac{1}{2} \dot{\theta}^{T} M(\theta) \dot{\theta}+P(\theta)-\frac{1}{2}{\dot{\theta_{R}}}^{T} M\left(\theta_{R}\right) \dot{\theta_{R}}-P\left(\theta_{R}\right)
$$

Where similarly to Proposition $1, P$ is a scalar function defined as follows:

$$
P(\theta)=U(\theta)+\frac{1}{2} \theta^{T} \Xi \theta-\int_{0}^{\theta}\left(\tau_{R}-\Gamma \dot{\theta}_{R}+\Xi \theta_{R}\right)^{T} d \theta
$$

Note that (22) becomes a particular case of (38) for $\theta_{R}=0$. Differentiating $P(\theta)$ in terms of $\theta$ gives:

$$
\begin{aligned}
\frac{\partial P(\theta)}{\partial \theta} & =G(\theta)+\Xi \theta-G\left(\theta_{R}\right)+\Xi \theta_{R}-F_{R} \\
F_{R} & =M\left(\theta_{R}\right) \ddot{\theta}_{R}+V_{m}\left(\theta_{R}, \dot{\theta}_{R}\right) \dot{\theta}_{R}-\Gamma \dot{\theta}_{R}
\end{aligned}
$$

Therefore

$$
\frac{\partial P(\theta)}{\partial \theta}=\left(\Xi-\Psi_{G}\left(\theta, \theta_{R}\right)\right) E-F_{R}
$$

Since $\Xi-\Psi_{G}\left(\theta, \theta_{R}\right)$ is positive definite, it can be shown that $P$ has an absolute minimum $P\left(\theta_{\min R}\right)$, function of the reference trajectory and given by $\frac{\partial P(\theta)}{\partial \theta}=0 . \theta_{\min R}$ verifies the following:

$$
\theta_{\min R}=\theta_{R}+\left(\Xi-\Psi_{G}\left(\theta, \theta_{R}\right)\right)^{-1} F_{R}
$$

Therefore $P(\theta) \geq P\left(\theta_{\min R}\right) \forall \theta$. Thus from (37), the Lyapunov-like function $L$ has a lower bound (not necessarily positive) given by:

$$
L \geq P\left(\theta_{\min R}\right)-\frac{1}{2} \dot{\theta}_{R}^{T} M\left(\theta_{R}\right) \dot{\theta}_{R}-P\left(\theta_{R}\right)
$$

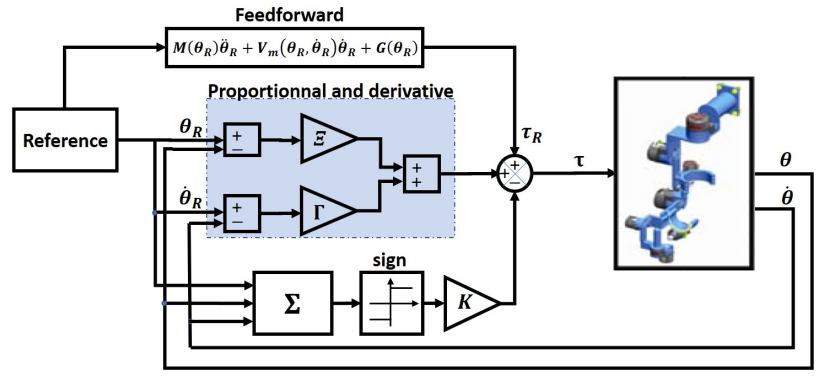

Fig. 4. Block Diagram of sliding mode control algorithm with model-based switching functions
Differentiating $L$ with respect to time gives the following:

$\dot{L}=\dot{\theta}^{T} \tau-\dot{\theta}_{R}^{T} \tau_{R}+\theta^{T} \Xi \dot{\theta}-\theta_{R}^{T} \Xi \dot{\theta}_{R}-\left(\tau_{R}-\Gamma \dot{\theta}_{R}+\Xi \theta_{R}\right)^{T} \dot{E}$

Using equation (36) in (43) when the sliding surface is reached:

$$
\begin{aligned}
\dot{L}= & \dot{\theta}^{T}\left(\tau_{R}-\Gamma \dot{E}-\Xi E\right)-\dot{\theta}_{R}^{T} \tau_{R}+\theta^{T} \Xi \dot{\theta}-\theta_{R}^{T} \Xi \dot{\theta}_{R} \\
& -\left(\tau_{R}-\Gamma \dot{\theta}_{R}+\Xi \theta_{R}\right)^{T} \dot{E}
\end{aligned}
$$

Simplifying (44) gives finally:

$$
\dot{L}=-\dot{E}^{T} \Gamma \dot{E}
$$

$\dot{L}$ is therefore negative semi-definite. Since $L$ has a lower bound, then as per [16], applying Barbalat's lemma implies that $\dot{L}$ converges to 0 , which then implies that $\dot{E}$ converges to 0 . Since $\Xi-\Psi_{G}\left(\theta, \theta_{R}\right)$ is positive definite, it can be proved that $E$ converges to 0 , which completes the proof of Proposition 2.

Note that constraint (35) is dependent on the reference trajectory $\theta_{R}$. It is however possible to formulate a constraint with an absolute lower bound for $\operatorname{Min}_{i}\left(\operatorname{Eig}_{i} \Xi\right)$ that is independent of the trajectory as per Proposition 3 below:

Proposition 3: The following holds:

$$
\operatorname{Max}_{i}\left(\operatorname{Eig}\left(\Psi_{G}\left(\theta, \theta_{R}\right)\right)\right) \leq \operatorname{Max}_{i}\left(\operatorname{Eig}\left(-J_{G}(\theta)\right)\right)
$$

Proof: Note $\lambda_{M a x}=\operatorname{Max}_{i}\left(\operatorname{Eig}\left(-J_{G}(\theta)\right)\right)$. In other words, $\lambda_{\text {Max }}$ is a constant absolute maximum of all varying eigenvalues of $-J_{G}(\theta)$. The following then holds:

$$
\begin{aligned}
\lambda_{\text {Max }} I_{n}-\Psi_{G}\left(\theta, \theta_{R}\right)= & \lambda_{\text {Max }} I_{n} \\
& -\left(-\int_{0}^{1} J_{G}\left(\theta_{R}+h\left(\theta-\theta_{R}\right)\right) d h\right) \\
= & \int_{0}^{1} \lambda_{\text {Max }} I_{n}-\left(-J_{G}\left(\theta_{R}+h(E)\right)\right) d h
\end{aligned}
$$

By construction, $\lambda_{M a x} I_{n}-\left(-J_{G}\left(\theta_{R}+h(E)\right)\right)$ is positive definite. Therefore $\int_{0}^{1} \lambda_{M a x} I_{n}-\left(-J_{G}\left(\theta_{R}+h(E)\right)\right) d h$ is positive definite, then (47) implies $\lambda_{M a x} I_{n}-\Psi_{G}\left(\theta, \theta_{R}\right)$ is positive definite, and thus (46) is straightforward.

From Proposition 3, a sufficient condition to meet constraint (35) can therefore be formulated as follows:

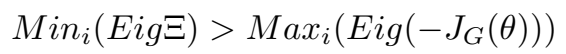

Remark 5: Note that the new constraint (48) represents a sufficient absolute condition on eigenvalues of $\Xi$ for any trajectory tracking within the robot's working space. The advantage of constraint (48) is that it is independent of the reference trajectory and holds for any trajectory within the robot's workspace. The disadvantage however is that the choice of the eigenvalues of matrix $\Xi$ might be overdimensioned to ensure constraint (48) is met. Also, as per Remark 2, one can choose as well in this case to compensate the gravity term in the torque control law rather than in the switching functions, which then removes the need of constraint (35). 


\section{Robustness Against Uncertainties}

In this section, model uncertainties are introduced with the model-based switching functions design approach. It is shown that the proposed approach compensates for uncertainties with the discontinuous gain in a similar way conventional sliding mode control does. The additional advantage of using modelbased switching functions is that only matched uncertainties are required to be compensated. Consider uncertainties on all the matrix terms of the robot model given by (1), and consider the following notations:

$\hat{M}(\theta), \hat{M}\left(\theta_{R}\right)$ (noted $\hat{M}$ and $\hat{M}_{R}$ ) are estimates of $M(\theta)$ and $M\left(\theta_{R}\right) ; \hat{V}_{m}(\theta, \dot{\theta}), \hat{V}_{m}\left(\theta_{R}, \dot{\theta}_{R}\right)$ (noted $\hat{V}_{m}$ and $\hat{V}_{m R}$ ) are estimates of $V_{m}(\theta, \dot{\theta}), V_{m}\left(\theta_{R}, \dot{\theta}_{R}\right)$ ); $\hat{G}(\theta), \hat{G}\left(\theta_{R}\right)$ (noted $\hat{G}$ and $\hat{G}_{R}$ ) are estimates of $G(\theta)$ and $G\left(\theta_{R}\right)$.

The switching function $\Sigma$ defined in (30) is then constructed by using the estimate matrices above as follows:

$$
\begin{aligned}
\Sigma= & \hat{M} \dot{\theta}-\hat{M}_{R} \dot{\theta}_{R}+\Gamma E+\Xi \int_{t_{0}}^{t} E d t+\int_{t_{0}}^{t}\left(\hat{G}-\hat{G}_{R}\right) d t \\
& -\int_{t_{0}}^{t}\left(\left(\hat{\sigma}+\hat{V}_{m}\right) \dot{\theta}-\left(\hat{\sigma}_{R}+\hat{V}_{m R}\right) \dot{\theta}_{R}\right) d t
\end{aligned}
$$

The time derivative of $\Sigma$ gives:

$$
\dot{\Sigma}=\hat{M} \ddot{\theta}+\hat{V}_{m} \dot{\theta}+\hat{G}-\hat{M}_{R} \ddot{\theta}_{R}-\hat{V}_{m R} \dot{\theta}_{R}-\hat{G}_{R}+\Gamma \dot{E}+\Xi E
$$

Introducing the torque input in $\dot{\Sigma}$ gives:

$$
\dot{\Sigma}=\tau+\tilde{M} \ddot{\theta}+\tilde{V}_{m} \dot{\theta}+\tilde{G}-\hat{\tau}_{R}+\Gamma \dot{E}+\Xi E
$$

with $\hat{\tau}_{R}=\hat{M}_{R} \ddot{\theta}_{R}+\hat{V}_{m R} \dot{\theta}_{R}+\hat{G}_{R}, \tilde{M}=\hat{M}-M(\theta), \tilde{V}_{m}=$ $\hat{V}_{m}-V_{m}(\theta, \dot{\theta})$ and $\tilde{G}=\hat{G}-G(\theta)$. Similarly to (31), choose the following control law:

$$
\tau=\hat{\tau}_{R}-\Gamma \dot{E}-\Xi E-K \operatorname{sign}(\Sigma)
$$

This leads to the following reaching law:

$$
\dot{\Sigma}=\tilde{M} \ddot{\theta}+\tilde{V}_{m} \dot{\theta}+\tilde{G}-K \operatorname{sign}(\Sigma)
$$

From (53), note that the term $\tilde{M} \ddot{\theta}+\tilde{V}_{m} \dot{\theta}+\tilde{G}$ exists because of the introduced uncertainties. Assuming this term is bounded, it is then possible to choose matrix $K$ elements larger than the upper bound of that term in order to ensure reaching the sliding surface. This uncertainty compensation is also a known procedure in conventional sliding mode control [16].

\section{EXPERIMENTAL APPLICATION ON ETS-MARSE}

This section describes the experimental setup of ETSMARSE exoskeleton prototype shown in the Fig. 7 (adapted from [14]). The real-time controller using the model-based switching functions algorithm was implemented on a National Instrument (NI) processing platform as detailed in Fig. 5. The NI processing platform comprises a NI-PXI 8081 dual-core controller card and a NI-PXI 7813-R FPGA card. Both cards are enclosed in a PXI-1031 chassis. As shown in Fig. 6, the NI-PXI 8081 controller executes at a time step of $5 \mathrm{~ms}$ the top-level sliding mode control algorithm with the model-based switching functions along with the inverse kinematics logic. The NI PXI-7813R FPGA card executes, on the other hand, the low-level current PI control loop at the faster sample time of 50 us. The NI PXI-7813R processes as well the joints position feedback via Hall-effect sensors. Finally, user-defined commands are sent to the robot through a host HMI PC using a LabView interface in which it is possible to select the controller type and perform online controller gain adjustments. The joints of ETS-MARSE are actuated by brushless DC motors Maxon EC-45 and EC-90 using harmonic drives to ensure high gear ratios while maintaining very good accuracy positioning [gear ratio for motor 1, 2 and 4: 120:1; gear ratio for motor 3, 5, 6 and 7: 100:1].

\section{A. Experimental Real-Time Results}

The experimental test cases that were executed on ETSMARSE are divided into two sets. The first set was executed without load and compares the model-based switching functions approach to conventional sliding mode control. The second set was performed by subject 1 (weight: $83 \mathrm{~kg}$, height: $1.83 \mathrm{~m}$ ) using the model-based switching functions approach, in order to test its robustness under normal loading conditions. The reference trajectory of ETS-MARSE tool tip in the Cartesian space for both sets was chosen to have a rectangle-like shape, which is representative of an actual arm mobility exercise performed by patients. The reference trajectory is depicted in dashed black in Fig. 8. The correspondent reference joint angles for the 7 axes in the joint space are shown in dashed black in Fig. 9. Numerical values of the sliding mode controller gains using conventional and model-based switching functions are shown in Table IV. For linear switching functions design, $K$ was tuned to ensure an acceptable level of robustness against disturbances. The relatively high values of $K$ were obtained as a result of low numerical values of $M$, which orders of magnitude are bounded to $10^{-3}$. For the model-based switching functions approach, since $M$ is eliminated in the discontinuous term of the torque control inputs, the values of $K$ are expected to be three orders of magnitude lower to ensure a similar level of robustness. For simplification purposes, the diagonal values of $K$ were therefore set at 0.25 . From the joint space reference

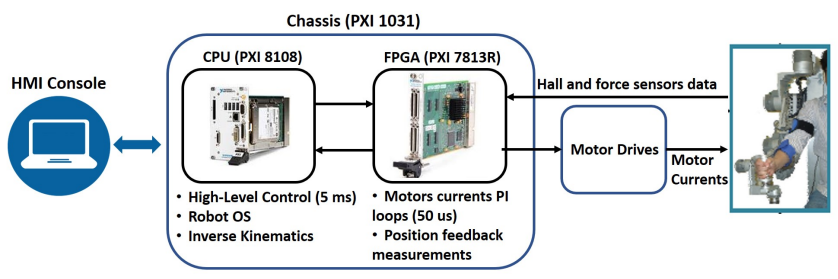

Fig. 5. Hardware Control Architecture Setup for ETS-MARSE

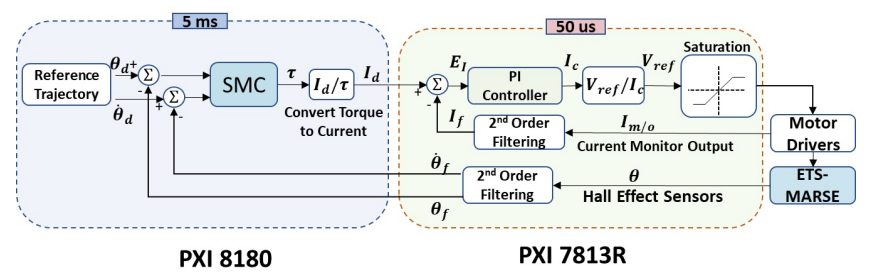

Fig. 6. Block Diagram of Control Algorithm Loops for ETS-MARSE 


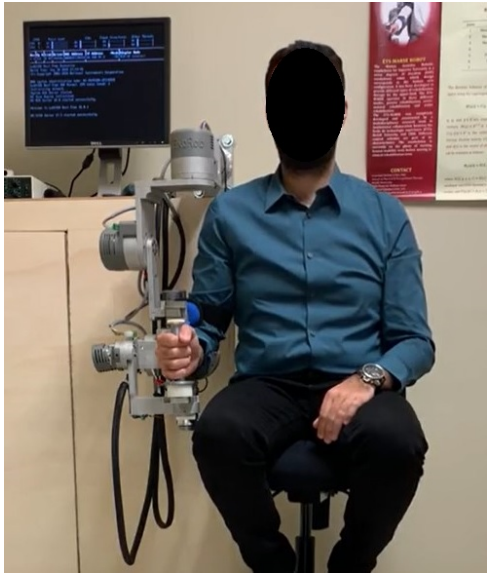

Fig. 7. ETS-MARSE Exoskeleton Prototype

TABLE IV

EXPerimental Control Parameters of ETS-MARSE

\begin{tabular}{c|cc}
\hline \hline & Parameter & Matrix Numerical Value \\
\hline Model-Based & $\Xi$ & $\operatorname{Diag} 7(25)$ \\
switching functions & $\Gamma$ & $\operatorname{Diag} 7(12.2)$ \\
(proposed) & $K$ & $\operatorname{Diag} 7(0.25)$ \\
\hline Linear switching & $\Lambda$ & $\operatorname{Diag} 7(15)$ \\
functions & $K$ & $10 * \operatorname{diag}(36,30,80,55,22,30,37)$ \\
\hline
\end{tabular}

trajectories, $\Psi_{G}\left(\theta, \theta_{R}\right)$ was symbolically derived for ETSMARSE and its upper eigenvalue limit numerically computed offline for the duration of the reference trajectory and showed to be inferior to 10 throughout this duration. Therefore by choosing all the eigenvalues of matrix $\Xi$ to be higher than 10 will guarantee as per (35) asymptotic stability of the tracking error to 0 for this particular trajectory. However, as Table IV shows, the diagonal elements numerical values of $\Xi$ were chosen to be 25 , which gives a good margin compared to the maximum eigenvalue limit of $-J_{G}(\theta)$ (numerically computed to be 21.32). The purpose of this choice is to ensure asymptotic convergence within the global work-space of ETS-MARSE regardless of the programmed reference trajectory, and to account as well for parameter uncertainties on the gravity matrix. Finally, the diagonal elements of matrix $\Gamma$ were set to 12.5 , which places a dominant pole in closed-loop varying between -2 and -2.5 , given the values of $\Xi$ and the numerical variation of $M$ along the trajectory.

Figures 10 and 11 display the results for the first set, and show the overlay of real-time performance of both modelbased and linear switching functions approaches on joint error tracking performance and joint torque inputs. From Figure 10 it can be seen that the proposed model-based switching functions approach features a better overall tracking accuracy on the joint angles. Table $\mathrm{V}$ gives a quantitative overview of the Peak and RMS tracking errors in the joint space. The modelbased switching functions yield an overall improvement of peak and RMS error compared to linear switching functions. The RMS improvement is particularly important for joints 2 and 4 (resp. 63.6\% and $87.44 \%$ ), which is visually supported

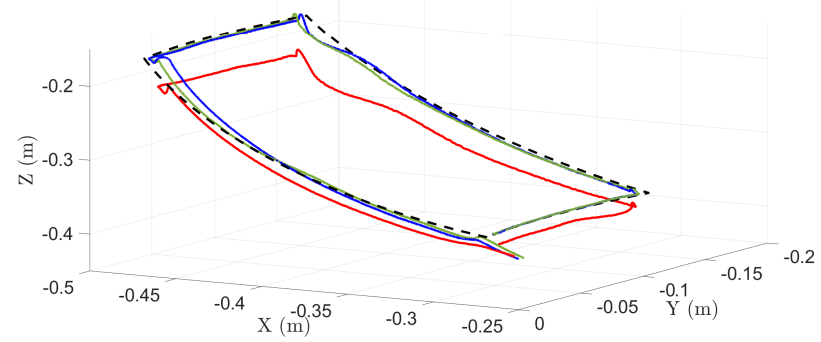

Fig. 8. Cartesian space reference trajectory (dashed black), no-load tracking performance for proposed method (blue), no-load conventional SM (red), loaded tracking performance for proposed method (green)

by the tracking superiority of the model-based switching functions approach over the conventional linear functions in the Cartesian space displayed in Fig. 8 . Table VI shows the measured total variation computed as per [38] on all torque control inputs. The total torque inputs variation is reduced by $20 \%$ up to $80 \%$ for axes 1 to 6 with the model-based switching functions approach, while staying comparable for wrist joint 7 . The torque inputs variation reduction is an important requirement for control design. Indeed, the practical implementation of a controller requires constraints reduction on the control inputs to help against premature failures of actuating components in the system, as well as avoiding nonmodeled fast dynamics behaviour in the closed-loop setup. Additionally, for the exoskeleton application, having reduced transient activity on the torques control inputs, reduces the vibration cues felt by the patient, and therefore ensures improved comfort and ergonomic requirements.
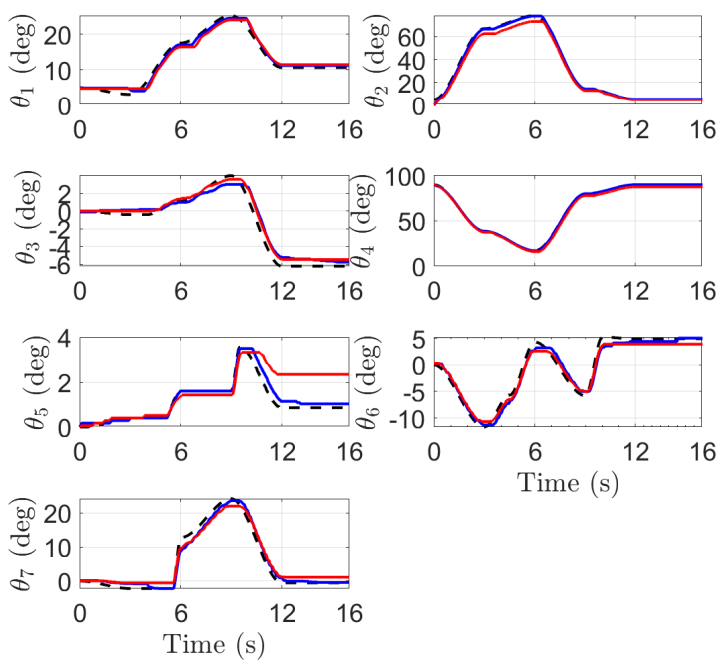

Fig. 9. Joint space reference trajectory (dashed black) and no-load tracking performance for proposed method (blue) and no-load conventional SM (red)

Figure 12 shows a zoomed-in time section of control torque input for axis 1 . Although the primary objective of the modelbased switching functions approach is not to address the chattering problem, the zoomed-in square of Figure 12 shows 

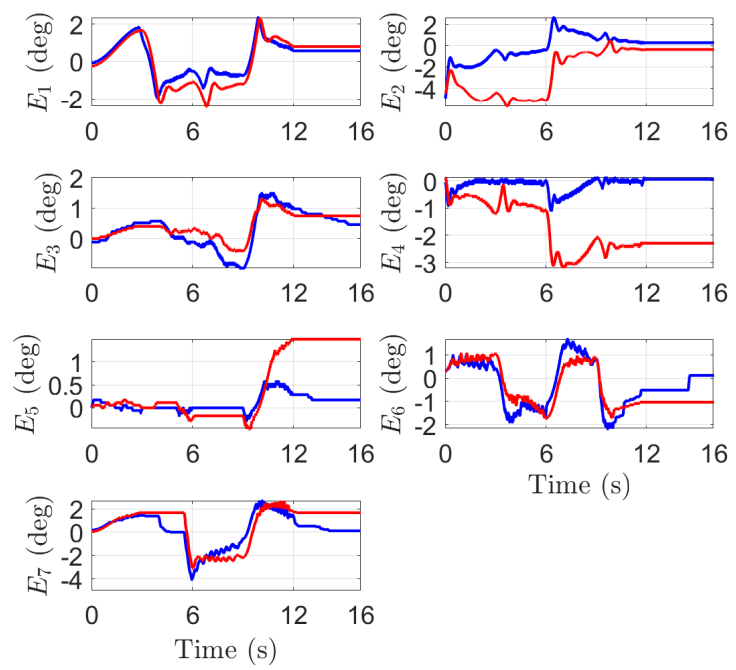

Fig. 10. No-load joint tracking errors for proposed method (blue) and no-load conventional SM (red)

at least $30 \%$ chattering level reduction on the torque control inputs due mainly to the inertia matrix compensation by the switching function, which leads to chattering decoupling on the control inputs. Transient dynamics with a 6 to $9 \mathrm{~Hz}$ frequency pattern also appears on the torque control input with conventional sliding mode control. These frequencies are caused by non-compensated model-based terms in the equivalent control input for the conventional approach.

Figures 13, 14 and 15 present the experimental results performed by subject-1 and feature the tracking performance and the torque inputs for the model-based switching functions approach under normal loading conditions. The tracking performance in this case is very good and remains within the boundaries of the no-load results, which demonstrates the robustness of the proposed approach under uncertain/nonmodeled loading conditions. The torques inputs variations are also comparable to the no-load results, with levels slightly higher especially for joints 1 to 4 , reflecting the addition of the arm weight of subject- 1 .

\section{CONCLUSION}

In this paper, a novel sliding mode approach with modelbased switching functions design was developed and experimentally tested on a 7-DOF exoskeleton robotic arm for trajectory tracking control. The main advantages of the modelbased switching functions design were highlighted. These advantages include a complete decoupling of chattering and transient constraints reduction on the torque control inputs, as well as increased robustness compared to conventional linear sliding functions, while ensuring better tracking performance. Future work will combine the use of model-based switching functions with existing chattering reduction techniques.

\section{REFERENCES}

[1] M. Wolfgang, V. Lukic, A. Sander, J. Martin, and D. Kupper, "Gaining robotics advantage," The Boston Consulting Group, June 2017.
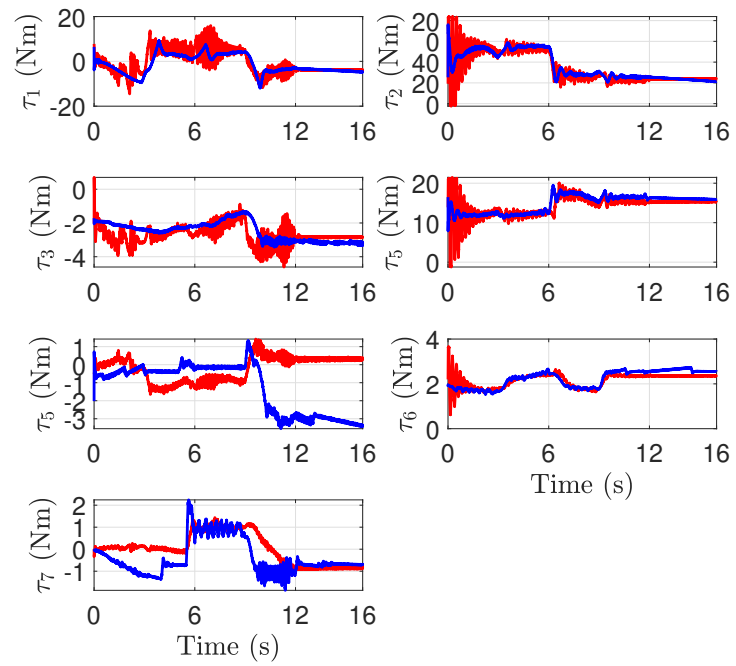

Fig. 11. No-load joint torque for proposed method (blue) and no-load conventional SM (red)

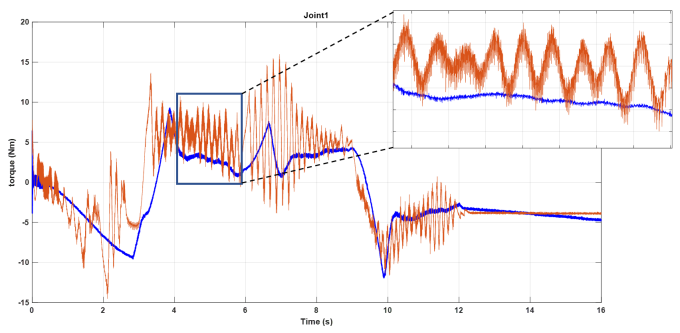

Fig. 12. Zoomed-in performance for axis 1 joint torque input, proposed method (blue) and conventional SM (red)
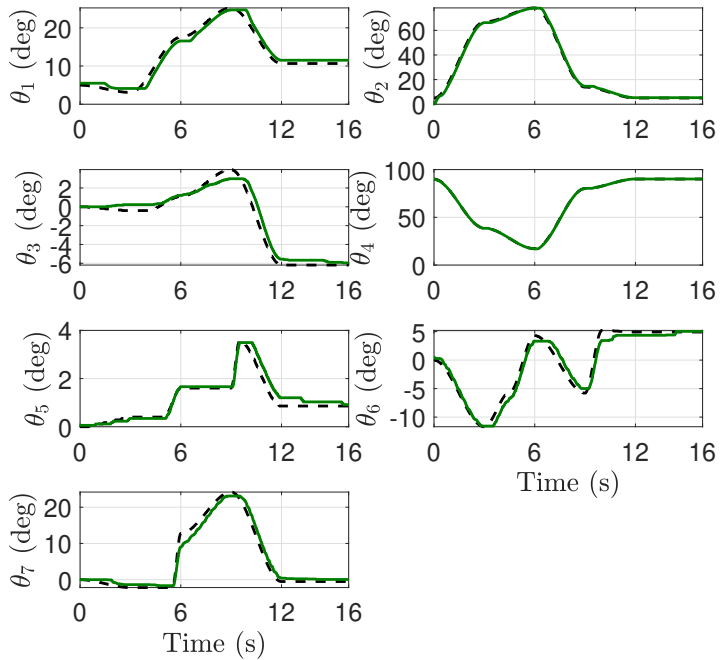

Fig. 13. Joint space reference trajectory (dashed black) and loaded tracking performance for proposed method (green) 
TABLE V

IMPROVEMENTS OF PROPOSED MODEL-BASED SWITCHING FUNCTIONS IN TRACKING ERROR PEAK AND RMS VALUES

\begin{tabular}{|c|c|c|c|c|c|c|}
\hline \multirow[b]{2}{*}{ Joint $(i)$} & \multicolumn{3}{|c|}{ Peak Error Value (degrees) } & \multicolumn{3}{|c|}{ RMS Error value (degrees) } \\
\hline & Conventional SMC & Proposed Approach & improvement (\%) & Conventional SMC & Proposed Approach & improvement $(\%)$ \\
\hline 1 & 2.46 & 2.29 & 6.98 & 1.2 & 0.92 & 23.53 \\
\hline 2 & 5.73 & 4.92 & 14.01 & 3.02 & 1.10 & 63.60 \\
\hline 3 & 1.32 & 1.49 & -13.05 & 0.59 & 0.69 & -16.98 \\
\hline 4 & 3.21 & 1.09 & 66.06 & 2.01 & 0.25 & 87.44 \\
\hline 5 & 1.49 & 0.57 & 61.54 & 0.85 & 0.20 & 76.59 \\
\hline 6 & 1.72 & 2.12 & -23.33 & 1.01 & 0.99 & 1.17 \\
\hline 7 & 3.09 & 4.01 & -29.64 & 1.77 & 1.40 & 20.98 \\
\hline
\end{tabular}
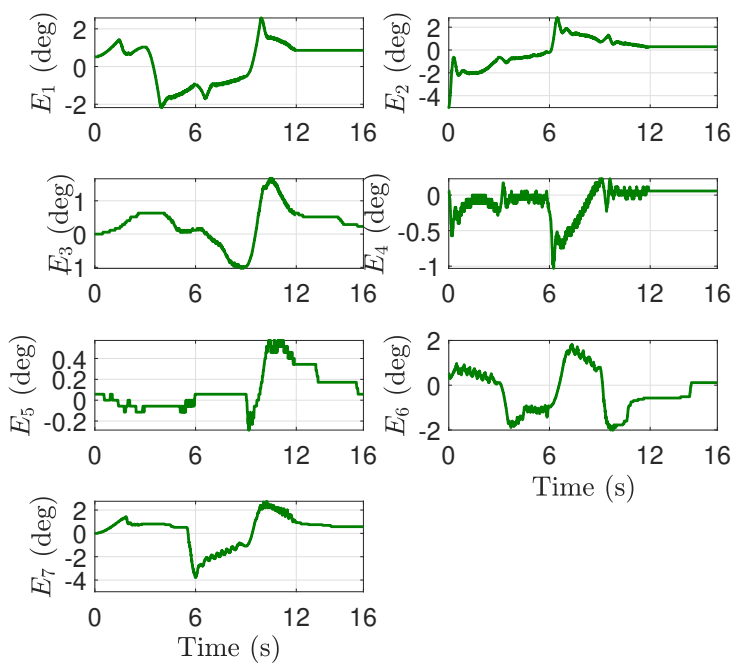

Fig. 14. Loaded joint tracking errors for proposed method
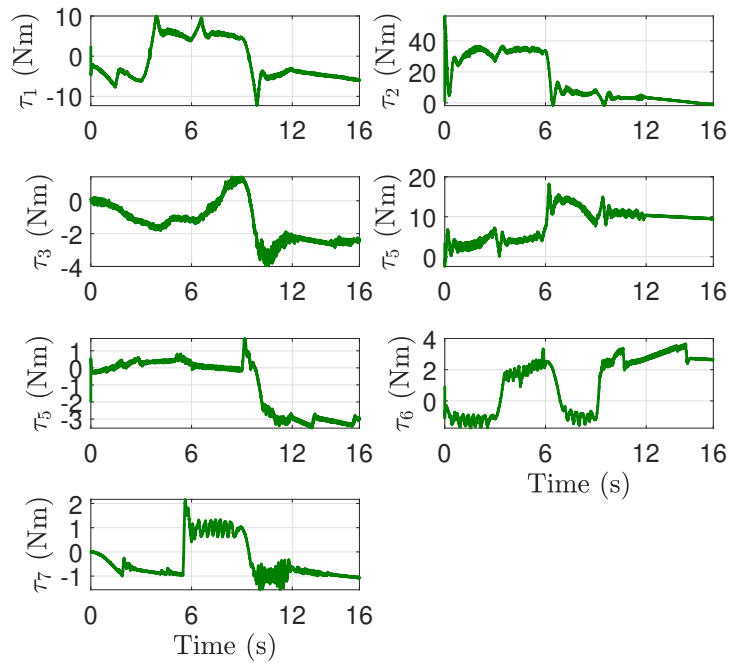

Fig. 15. Loaded joint torque for proposed method
TABLE VI

IMPROVEMENTS OF PROPOSED MODEL-BASED SWITCHING FUNCTIONS IN TORQUE INPUTS TOTAL VARIATION

\begin{tabular}{cccc}
\hline \hline \multirow{2}{*}{ Joint $(i)$} & \multicolumn{3}{c}{ Measured Torques Control Input Total Variance (Nm) } \\
\cline { 2 - 4 } & $\begin{array}{c}\text { Conventional } \\
\text { SMC }\end{array}$ & $\begin{array}{c}\text { Proposed } \\
\text { Approach }\end{array}$ & improvement $(\%)$ \\
\hline 1 & 1572.0 & 334.5 & 78.72 \\
2 & 2766.0 & 894.8 & 67.65 \\
3 & 492.7 & 212.8 & 56.81 \\
4 & 1315.0 & 566.6 & 56.91 \\
5 & 152.6 & 121.1 & 20.64 \\
6 & 233.6 & 113.1 & 51.58 \\
7 & 80.24 & 84.19 & -4.92 \\
\hline \hline
\end{tabular}

[2] Exoskeleton Market Size, Share Trends Analysis Report By Technology Type (Mobile, Stationary), By Technology Drive Type, By End User, By Region, And Segment Forecasts. Grand View Research, 2020.

[3] Global Wearable Robotic Exoskeleton Market, by Value and Volume: Focus on Mode of Operation, End User, Application, Material Type, and Limb Type. Analysis Forecast 2018-2028, BIS research, 2018.

[4] J. C. Perry, J. Rosen, and S. Burns, "Upper-limb powered exoskeleton design," IEEE/ASME Transactions on Mechatronics, vol. 12, no. 4, pp. 408-417, Aug 2007.

[5] N. Tsagarakis and D. G. Caldwell, "Development and control of a 'soft-actuated' exoskeleton for use in physiotherapy and training," Autonomous Robots, vol. 15, no. 1, pp. 21-33, Jul 2003.

[6] A. Frisoli, F. Salsedo, M. Bergamasco, B. Rossi, and M. C. Carboncini, "A force-feedback exoskeleton for upper-limb rehabilitation in virtual reality," Applied Bionics and Biomechanics, vol. 6, no. 2, pp. 115-126, 2009.

[7] Z. Li, B. Huang, Z. Ye, M. Deng, and C. Yang, "Physical human-robot interaction of a robotic exoskeleton by admittance control," IEEE Transactions on Industrial Electronics, vol. 65, no. 12, pp. 9614-9624, Dec 2018.

[8] A. Gupta and M. K. O'Malley, "Design of a haptic arm exoskeleton for training and rehabilitation," IEEE/ASME Transactions on Mechatronics, vol. 11, no. 3, pp. 280-289, June 2006.

[9] L. Lucas, M. DiCicco, and Y. Matsuoka, "An emg-controlled hand exoskeleton for natural pinching," Journal of Robotics and Mechatronics, vol. 16, no. 5, pp. 482 - 488, January 2004

[10] W. Yu and J. Rosen, "A novel linear pid controller for an upper limb exoskeleton," in 49th IEEE Conference on Decision and Control (CDC), 2010, pp. 3548-3553.

[11] G. P. Incremona, A. Ferrara, and L. Magni, "Mpc for robot manipulators with integral sliding modes generation," IEEE/ASME Transactions on Mechatronics, vol. 22, no. 3, pp. 1299-1307, 2017.

[12] M. Rahman, T. Kittel-Ouimet, M. Saad, K. Jean-Pierre, and P. Archambault, "Development and control of a robotic exoskeleton for shoulder, elbow and forearm movement assistance," Applied Bionics and Biomechanics, vol. 9, pp. 275-292, 072012.

[13] M. Rahman, C. Ochoa Luna, M. Rahman, M. Saad, and P. Archambault, "Force-position control of a robotic exoskeleton to provide upper 
extremity movement assistance," International Journal of Modelling, Identification and Control, vol. 21, pp. 390-400, 012014.

[14] M. Rahman, C. Ochoa Luna, M. Saad, and P. Archambault, "Motion control of an exoskeleton robot using electromyogram signals," Advances in Robotics, Mechatronics and Circuits, pp. 27-33, 2014.

[15] C. O. Luna, M. H. Rahman, M. Saad, P. S. Archambault, and S. B. Ferrer, "Admittance-based upper limb robotic active and activeassistive movements," International Journal of Advanced Robotic Systems, vol. 12, no. 9, p. 117, 2015.

[16] J. J. Slotine and W. Li, Applied Nonlinear Control. NJ, Englewood Cliffs: Prentice-Hall, 1991.

[17] S. Roy, S. B. Roy, J. Lee, and S. Baldi, "Overcoming the underestimation and overestimation problems in adaptive sliding mode control," IEEE/ASME Transactions on Mechatronics, vol. 24, no. 5, pp. 20312039, Oct 2019.

[18] Y. Wang, W. Zhou, J. Luo, H. Yan, H. Pu, and Y. Peng, "Reliable intelligent path following control for a robotic airship against sensor faults," IEEE/ASME Transactions on Mechatronics, pp. 1-1, 2019.

[19] R. Temporelli, M. Boisvert, and P. Micheau, "Control of an electromechanical clutch actuator using a dual sliding mode controller: Theory and experimental investigations," IEEE/ASME Transactions on Mechatronics, vol. 24, no. 4, pp. 1674-1685, Aug 2019.

[20] Weibing Gao and J. C. Hung, "Variable structure control of nonlinear systems: a new approach," IEEE Transactions on Industrial Electronics, vol. 40, no. 1, pp. 45-55, Feb 1993.

[21] O. Camacho, R. Rojas, and W. Garcia, "Variable structure control applied to chemical processes with inverse response," ISA Transactions, vol. 38, no. 1, pp. $55-72,1999$.

[22] C. J. Fallaha, M. Saad, H. Y. Kanaan, and K. Al-Haddad, "Slidingmode robot control with exponential reaching law," IEEE Transactions on Industrial Electronics, vol. 58, no. 2, pp. 600-610, Feb 2011.

[23] A. Levant, "Sliding order and sliding accuracy in sliding mode control," International Journal of Control, vol. 58, no. 6, pp. 1247-1263, 1993.

[24] F. Hamerlain, K. Achour, T. Floquet, and W. Perruquetti, "Trajectory tracking of a car-like robot using second order sliding mode control," in 2007 European Control Conference (ECC), July 2007, pp. 4932-4936.

[25] G. Bartolini, A. Ferrara, E. Usai, and V. I. Utkin, "On multi-input chattering-free second-order sliding mode control," IEEE Transactions on Automatic Control, vol. 45, no. 9, pp. 1711-1717, Sep. 2000.

[26] V. Parra-Vega and G. Hirzinger, "Chattering-free sliding mode control for a class of nonlinear mechanical systems," International Journal of Robust and Nonlinear Control, vol. 11, no. 12, pp. 1161-1178, 2001.

[27] J. Sivaramakrishnan, M. Agwan, and L. Dewan, "A smart higher order sliding mode control of rigid articulated robotic manipulator with passive joints," International Journal of Modelling Identification and Control, vol. 23, pp. 260-266, 062015.

[28] J. A. Moreno and M. Osorio, "A lyapunov approach to second-order sliding mode controllers and observers," in 2008 47th IEEE Conference on Decision and Control, Dec 2008, pp. 2856-2861.

[29] T. Floquet and J. P. Barbot, "Super twisting algorithm-based step-by-step sliding mode observers for nonlinear systems with unknown inputs," International Journal of Systems Science, vol. 38, no. 10, pp. 803-815, 2007.

[30] T. Gonzalez, J. A. Moreno, and L. Fridman, "Variable gain supertwisting sliding mode control," IEEE Transactions on Automatic Control, vol. 57, no. 8, pp. 2100-2105, Aug 2012.

[31] Y. Tang, "Terminal sliding mode control for rigid robots," Automatica, vol. 34, no. 1, pp. $51-56,1998$.

[32] L. Dai, Y. Yu, D. Zhai, T. Huang, and Y. Xia, "Robust model predictive tracking control for robot manipulators with disturbances," IEEE Transactions on Industrial Electronics, pp. 1-1, 2020.

[33] J. J. Craig, Introduction to Robotics Mechanics and Control, 3rd ed. Pearson Education, Inc., 2005.

[34] C. Fallaha and M. Saad, "Model-based sliding functions design for sliding mode robot control," International Journal of Modelling, Identification and Control, vol. 30, p. 48, 012018.

[35] M. W. Spong, S. Hutchinson, and M. Vidyasagar, Robot Modeling and Control. John Wiley and Sons, Inc., 2005.

[36] M. Comenetz, Calculus: The Elements. World Scientific, 2002.

[37] P. Tomei, "Adaptive pd controller for robot manipulators," IEEE Transactions on Robotics and Automation, vol. 7, no. 4, pp. 565-570, Aug 1991.

[38] S. Mondal and C. Mahanta, "Adaptive second order terminal sliding mode controller for robotic manipulators," Journal of the Franklin Institute, vol. 351, no. 4, pp. 2356 - 2377, 2014.

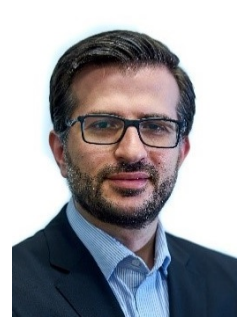

Charles Fallaha obtained his Bachelor's in Electrical and Mechanical Engineering from the École Supérieure d'Ingénieurs de Beyrouth (ESIB) and his Master's in Electrical Engineering from the École de technologie supérieure (ÉTS). He has more than 15 years of professional experience as an engineer, during which he has filled several engineering positions in the industry. He has also been a parttime Lecturer at the ÉTS where he currently teaches robotics modelling, programming and control. $\mathrm{He}$ is currently completing his $\mathrm{PhD}$ in mechatronics and nonlinear robotics control. His main research interests include nonlinear and intelligent control algorithms applied to robotic systems.

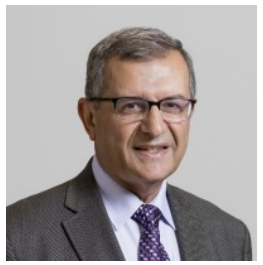

Maarouf Saad received the B.S. and M.S. degrees in electrical engineering from École Polytechnique of Montreal, Montreal, QC, Canada, in 1982 and 1984, respectively, and the Ph.D. degree in electrical engineering from McGill University, Montreal, in 1988. In 1987, he joined École de Technologie Supérieure, Montreal, where he is currently teaching control theory and robotics courses. His research is mainly in nonlinear control and optimization applied to robotics and flight control system.

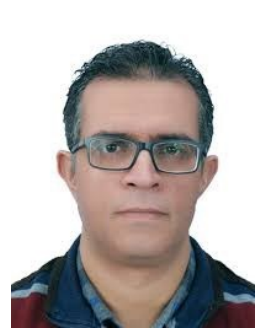

Jawhar Ghommam received the B.Sc. degree in computer and control engineering from the National Institute and Applied Sciences and Technology, Tunis, Tunisia, in 2003, the DEA (M.Sc.) degree from the University of Montpelier, Laboratoire d'Informatique, Robotique et Microlectronique, Montpellier, France, in 2004, and the joint Ph.D.degree in control engineering from the National Engineering School of Sfax, Sfax, Tunisia, and the University of Orleans, Orléans, France, in 2008. From 2008 to 2017, he was with the National Institute of Applied Sciences and Technology, where he held a tenured Associate Professor position with the Department of Physics and Instrumentation. In 2018, he joined the Department of Electrical and Computer Engineering, Sultan Quaboos University (SQU), Muscat, Oman. He is a member of the Control and Energy Management Lab and also an Associate Researcher with the GREPCI-Lab, Ecole de Technologie Superieure, Montreal, QC, Canada. His research interests include fundamental motion control concepts for nonholonomic/underactuated vehicle systems, nonlinear and adaptive control, intelligent and autonomous control of networked unmanned systems, team cooperation, consensus achievement, and sensor networks. Dr. Ghommam serves as a regular Referee and Associate Editor for many international journals in the filed of control and robotics.

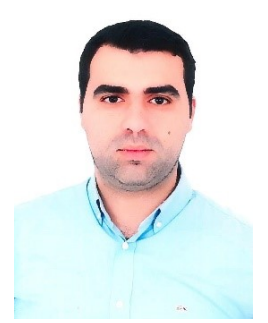

Yassine Kali Yassine Kali was born in Fez, Morocco. He received his B.Eng. and M.Sc. degrees in Electrical Engineering from the Faculté des Sciences et techniques, Fez, Morocco, respectively in 2011 and 2013. In january 2018, he received his Ph.D degree in the field of robotics and control systems from Ecole Mohammadia d'Ingénieurs (EMI), University of Mohammed V, Rabat, Morocco.

Since may 2018, he joined the GREPCI Laboratory of École de Technologie Supérieure (Montreal, Canada) as Postdoctoral Research Fellow. His research interests include robotics, multiphase drives, robust nonlinear control, sliding mode theory and renewable energy conversion systems. 